\title{
Long-Term Effect of Mediterranean-Style Diet and Calorie Restriction on Biomarkers of Longevity and Oxidative Stress in Overweight Men
}

\author{
Katherine Esposito, Carmen Di Palo, Maria Ida Maiorino, Michela Petrizzo, \\ Giuseppe Bellastella, Isabella Siniscalchi, and Dario Giugliano
}

Chair and Division of Metabolic Diseases, Second University of Naples, Piazza L. Miraglia, 80138 Napoli, Italy

Correspondence should be addressed to Katherine Esposito, katherine.esposito@unina2.it

Received 17 September 2010; Accepted 12 November 2010

Academic Editor: Demosthenes Panagiotakos

Copyright (c) 2011 Katherine Esposito et al. This is an open access article distributed under the Creative Commons Attribution License, which permits unrestricted use, distribution, and reproduction in any medium, provided the original work is properly cited.

\begin{abstract}
We report the effects of a Mediterranean-style diet, with or without calorie restriction, on biomarkers of aging and oxidative stress in overweight men. 192 men were randomly assigned to either a Mediterranean-style diet or a conventional diet. The intervention program was based on implementation of a Mediterranean dietary pattern in the overweight group (MED diet group), associated with calorie restriction and increased physical activity in the obese group (lifestyle group). Both groups were compared with participants in two matched control groups (advice groups). After 2 years, there was a significant difference in weight loss between groups, which was $-14 \mathrm{~kg}(95 \% \mathrm{CI}-20$ to -8$)$ in lifestyle groups and $-2.0 \mathrm{~kg}(-4.4$ to 0$)$ in the advice groups, with a difference of $-11.9 \mathrm{~kg}(\mathrm{CI}-19$ to $-4.7 \mathrm{~kg}, P<.001)$; moreover, there was a significant difference between groups at 2 years for insulin $(P=.04), 8$-iso-PGF2 $\alpha(P=.037)$, glucose $(P=.04)$, and adiponectin $(P=.01)$. Prolonged adherence to a Mediterranean-style diet, with or without caloric restriction, in overweight or obese men is associated with significant amelioration of multiple risk factors, including a better cardiovascular risk profile, reduced oxidative stress, and improved insulin sensitivity.
\end{abstract}

\section{Introduction}

Data from observational and cohort studies indicate that longevity is greater in individuals adhering to healthy lifestyle practices [1] or in those with a favorable level of risk factors $[2,3]$. Because of the limitations in feasibility for randomized trials to address the long-term effects of lifestyle on clinical outcomes, clinicians must rely mainly on a combination of epidemiological investigations and short-term clinical trials with intermediate end points. Whether long-term lifestyle changes add years to life and slow aging is not yet known. Some recent findings suggest that calorie restriction may have a positive effect on surrogate markers of longevity, including fasting insulin level and body temperature, as well as markers of oxidative stress [4]. Although the data were based on small number of subjects and limited followup (up to six months), the results were in line with the hypothesis that decreasing caloric intake by $30 \%$ or more in young or middle-aged laboratory animals prevents or retards age-related chronic diseases and significantly prolongs maximal life span $[5,6]$. The quality of diet may also be important, as secondary prevention trials have indicated that Mediterranean-style diets reduced rates of heart disease and cardiovascular mortality in people with established coronary diseases [7, 8]. Moreover, increasing evidence suggests that diet may also be important in modulating inflammation $[9,10]$. We are not aware of any trials assessing the longterm effect of the quality of diet on markers of longevity or oxidative stress in primary prevention. We report the effects of a Mediterranean-style diet, with or without calorie restriction, on biomarkers of aging and oxidative stress in overweight men.

\section{Methods}

2.1. Study Participants. Men were identified in our database of subjects participating in randomized controlled trials evaluating the effect of lifestyle changes $[11,12]$ and conducted 
from October 2000 to January 2004. Men included in the present analysis were obese subjects with erectile dysfunction [11] or overweight subjects with metabolic syndrome [12] and had to have a complete follow-up in the respective study trial.

Participants were recruited from the outpatient practices of the Teaching Hospital of the Second University of Naples, Italy, and included persons of 18 years of age and older with a body mass index (BMI) of $26 \mathrm{~kg} / \mathrm{m}^{2}$ or greater. The subjects were sedentary (less than one hour per week of physical activity) with no evidence of participation in diet reduction programs and with a stable weight $( \pm 1 \mathrm{~kg})$ within the last 6 months. The exclusion criteria were diabetes mellitus, impaired renal function (serum creatinine level greater than $1.5 \mathrm{mg} / \mathrm{dL}$ ), hepatic disease, cardiovascular disease, psychiatric problems, a history of alcohol abuse (at least $500 \mathrm{~g}$ alcohol/week in the last year), and use of any medication. Subjects with the metabolic syndrome had to have three or more of the criteria recommended by the Adult Treatment Panel [13]. The study was approved by the institutional committee of ethical practice of our institution, and all the study subjects gave informed written consent.

2.2. Interventions. The intervention program was based on implementation of a Mediterranean dietary pattern in the overweight group (MED diet group), combined with calorie restriction and increased physical activity in the obese group (lifestyle group). Both groups were compared with two matched control groups (advice groups). Subjects were randomly assigned to either the intervention or advice groups using a computer-generated random number sequence. The program involved education on reducing dietary calories, personal goal setting, and self-monitoring (food diaries) through a series of monthly small-group sessions. The recommended composition of the dietary regimen was the following: carbohydrates $50 \%$ to $60 \%$, proteins $15 \%$ to $20 \%$, total fat $\leq 30 \%$, saturated fat $<10 \%$; subjects were also advised to increase consumption of fruits, vegetables, nuts, and whole grains daily, and to increase the consumption of olive oil. Patients were in the program for 24 months and had monthly sessions with the nutritionist for the first year and bimonthly sessions for the second year. Compliance with the program was assessed by attendance at the meetings and completion of the diet diaries. Subjects in the lifestyle group also received guidance on increasing their level of physical activity, mainly walking for a minimum of 30 minutes per day, but also swimming or aerobic ball games. Subjects in the advice groups were given general oral and written information about healthy food choices both at baseline and at subsequent visits, but no specific individualized programs were offered to them.

2.3. Outcome Measures. Outcome measures were recorded at one and two years. The primary end point was change from baseline in insulin and 8 -iso- $\mathrm{PGF}_{2 \alpha}$ between intervention and advice groups. Secondary analyses included the changed from baseline in body weight, total cholesterol, blood pressure, glucose, and adiponectin.
Height and weight were recorded with participants wearing lightweight clothing and no shoes using a Seca 200 scale with attached stadiometer (Seca, Hamburg, Germany). Twenty-four-hour nutrient intakes were calculated with food composition tables and patients' weekly diet diaries. All subjects were asked to complete for 3-day food record and to record occupational, household, and leisure time physical activity, to assess dietary adherence and exercise activity. Estimation of insulin sensitivity in the fasting state was assessed with HOMA (homeostasis model assessment) and calculated with the following formula: fasting plasma glucose $(\mathrm{mmol} / \mathrm{L}) \times$ fasting serum insulin $(\mu \mathrm{U} / \mathrm{mL}) / 25$, as described by Matthews et al. [14]. Assays for serum total and highdensity lipoprotein cholesterol, triglycerides, and glucose levels were performed in the hospital's chemistry laboratory. Plasma insulin levels were assayed by radioimmunoassay (Ares, Serono). Serum samples for adiponectin were stored at $-80^{\circ} \mathrm{C}$ until assayed in duplicate using a high-sensitive, quantitative sandwich enzyme assay (Quantikine HS, R\&D Systems, Minneapolis, MN). Plasma total 8-iso-PGF $2 \alpha$ levels were assessed by enzyme immunoassay (Assay Design Inc., Indianapolis, IN).

2.4. Statistical Analysis. Data are presented as mean \pm SD unless otherwise stated. Unpaired (intervention versus advice) and paired (before versus after treatment) Student's $t$-tests were performed. The effect of treatments were tested by means of paired $t$-tests and a Wilcoxon matched test. The results of statistical analysis were also confirmed with ANOVA for repeated measures. Results were considered significant with two-tailed $P<.05$. All analyses were conducted using SPSS version 11.1 (SPSS Inc, Chicago, Ill).

\section{Results}

A total of 192 men enrolled in the study, 98 men in the intervention groups (46 MED diet, 52 lifestyle) and 94 men (44 MED diet, 50 lifestyle) in the advice groups. Both intervention groups were comparable with the corresponding advice groups, including the number of components of the metabolic syndrome, and relatively healthy. The prevalence of smokers was similar in the 2 groups: $27 \%$ in the intervention group and $31 \%$ in the control group $(P=.34)$. Moreover, baseline data showed no important difference in the nutrient intake between the two groups (data not shown). After 2 years, subjects on the intervention groups consumed a greater percentage of calories from complex carbohydrates, polyunsaturated, and monounsaturated fat, had higher fiber intake, a lower ratio of omega- 6 to omega- 3 fatty acids, as well as lower energy consumption, saturated fat, and cholesterol intake than controls. Total fruit, vegetable, nuts, and whole grain intakes and olive oil consumption were significantly higher in the intervention groups. The level of physical activity increased more in the intervention group (from 39 [10] to 156 [30] $\mathrm{min} / \mathrm{wk}$ ) than in the control group (from 41 [9] to 74 [28] $\mathrm{min} / \mathrm{wk} ; P<.001$ ).

After 2 years, there was a significant difference in weight loss between groups, which was $-14 \mathrm{~kg}(95 \% \mathrm{CI}-20$ to -8$)$ 


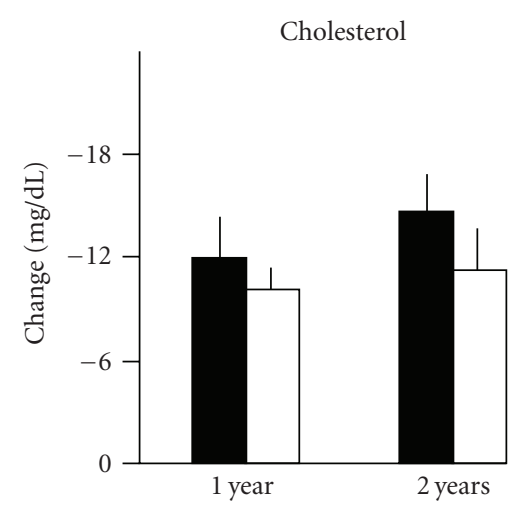

(a)

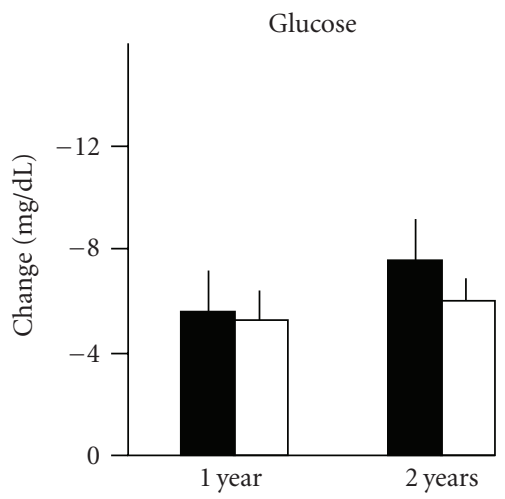

(d)

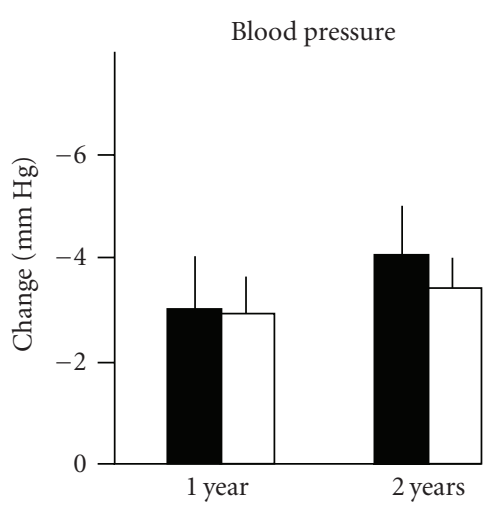

(b)

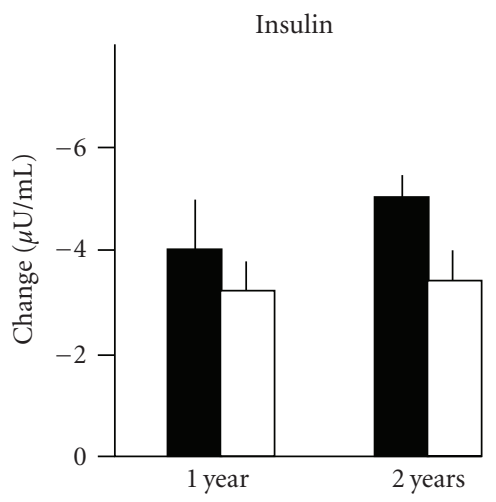

(e)

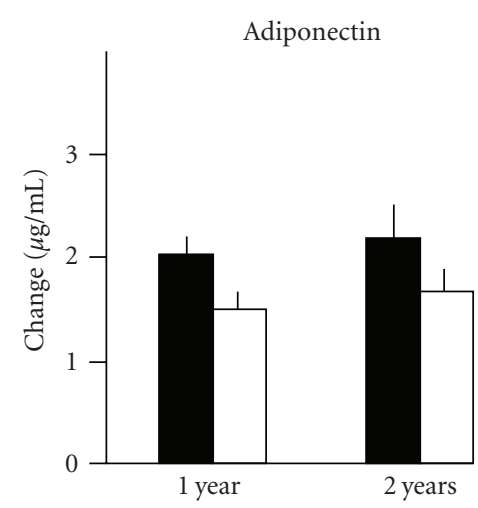

(c)

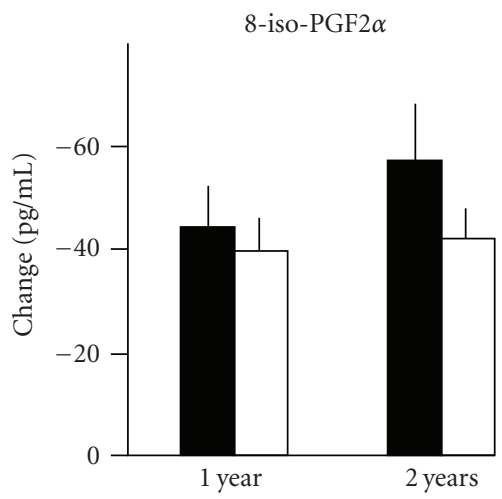

(f)

FIGURE 1: Changes in fasting plasma total cholesterol, glucose, insulin, mean blood pressure, insulin, adiponectin, and 8-iso-PGF $2 \alpha$ levels at year 1 and year 2 in subjects of intervention groups (black columns: lifestyle, white columns: MED diet). All parameters represent the net effect (intervention group, advice group) of treatments and were significantly reduced from baseline values at year 1 and year 2 in both groups.

in lifestyle groups and $-2.0 \mathrm{~kg}(-4.4$ to 0$)$ in the advice groups, with a difference of $-11.9 \mathrm{~kg}(\mathrm{CI}-19$ to $-4.7 \mathrm{~kg}, P<$ $.001)$. There was a significant association between change in body weight and HOMA $(r=0.43, P=.01)$, as well as between waist and HOMA $(r=0.37, P=.01)$.

Figure 1 shows the effect of treatments (change in intervention groups minus change in advice groups) on selected parameters at one year and two years. The effect of treatment was sustained as there was no trend to decrease at 2 years; moreover, there was a significant difference between groups (MED diet versus lifestyle) at 2 years for insulin $(P=.04), 8$-iso- $\mathrm{PGF}_{2 \alpha}(P=.037)$, glucose $(P=.04)$, and adiponectin $(P=.01)$ favouring lifestyle. There was no significant interaction of groups (intervention and advice) per time (years). The differences remained still significant after adjustment for the basal body weight.

\section{Discussion}

Our findings indicate that prolonged adherence to a Mediterranean-style diet, with or without caloric restriction, in overweight men resulted in reduced levels of biomarkers of ageing (insulin and glucose), oxidative stress (8-iso-PGF $2 \alpha$ ), and cardiovascular risk (cholesterol and blood pressure) and also produced a rise in circulating level of adiponectin, a protective factor against atherosclerosis and insulin resistance [15]. Long-term intervention studies of lifestyle changes on hard outcomes are unlikely to be performed. However, some of the surrogate markers evaluated in the present analysis are associated with extended life in observational studies [1-3].

Despite the fact that the role of oxidant stress in the pathogenesis of atherosclerosis is a hotly debated issue, in part because of a presumed failure of antioxidants to prevent the disease, current evidence suggests that isoprostanes represent a biomarker that has the potential to be of great importance in the assessment of human atherosclerotic cardiovascular disease. In particular, 8-iso-prostaglandin $\mathrm{F}_{2 \alpha}$ $\left(8\right.$-iso- $\left.\mathrm{PGF}_{2 \alpha}\right)$ is considered a reliable indicator of oxidative stress in vivo $[16,17]$. Recent evidence suggests that their quantification may represent an independent marker of atherosclerotic risk [18]. Moreover, increasing evidence suggests an association between oxidative stress and obesity or insulin resistance. Keaney et al. [19] reported an association between increasing body mass index and increasing systemic oxidant stress; in nearly 3000 patients involved in the Framingham Heart Study, the authors showed enhanced IsoP formation in men and women, strongly associated with 
increasing body mass index. Data from 2,002 nondiabetic subjects of the community-based Framingham Offspring Study indicate that systemic oxidative stress is associated with insulin resistance in individuals at average or elevated risk of diabetes (metabolic syndrome or IFG) even after accounting for BMI [20].

Short-term (up to six months) caloric restriction in overweight men and women produced significant body weight reduction associated with decreased DNA damage, considered a surrogate marker of oxidative stress [4]. The oxidative stress hypothesis of aging finds support from animal studies demonstrating reduced oxidative damage in longer-surviving, calorie-restriced rodents [21]. On the other hand, individuals at high cardiovascular risk who improved their diet toward a Mediterranean dietary pattern showed significant reductions in cellular lipid levels and LDL oxidation [22]. This recent observation adds evidence to the intriguing hypothesis that one important effect of Mediterranean-style diets in prolonging life $[23,24]$ may be associated with reduced oxidative stress and inflammation $[9,10]$.

Adiponectin plays an important role in modulating both insulin sensitivity and concentrations of circulating plasma glucose and nonesterified fatty acids. A lower concentration of circulating plasma adiponectin seems to be a good predictor of reduced insulin sensitivity and increased risk of type 2 diabetes [25]. In 987 diabetic women from the Nurses' Health Study with no history of cardiovascular disease, close adherence to a Mediterranean-type diet associated with higher adiponectin concentrations [26]. Of the several components of the Mediterranean dietary pattern score, alcohol, nuts, and whole grains show the strongest association with adiponectin concentrations. In insulin-resistant offspring of obese type 2 diabetic patients, a Mediterranean-style diet rich in monounsaturated fats (23\% MUFA) improved insulin sensitivity, and this was associated with increased postprandial adiponectin mRNA gene expression in peripheral adipose tissue [27].

Interestingly enough, many of the parameters that are positively affected in the intervention groups are important risk factors for healthy survival in a large group of middleaged men, including overweight, and high glucose, triglyceride, and blood pressure levels [3]. Given the difficulty of adopting a calorie restricted diet in the long-run, it seems important that such changes may be obtained, although to a lesser degree, with improved quality of diet. The public health burden of chronic diseases related to unhealthy diet is huge and on the rise, and argues for concomitant public health strategies and policies that affect entire populations.

In conclusion, prolonged adherence to a Mediterraneanstyle diet, with or without caloric restriction, in overweight or obese men is associated with significant amelioration of multiple risk factors, including a better cardiovascular risk profile (less blood pressure, less total cholesterol and triglycerides, higher HDL-cholesterol), reduced oxidative stress (less iso-8- $\mathrm{PGF}_{2 \alpha}$ ), and improved insulin sensitivity (reduced HOMA and increased adiponectin levels). These changes are amplified when MED diet is associated with calorie restriction and increased physical activity.
As several potential risk factors for healthy and long survival in men are modifiable, common approaches that target multiple risk factors simultaneously and approaches that enhance insulin sensitivity may improve the probability of better health at older ages [3]. The choice of healthy diets, as the Mediterranean-style diet is thought to be, is critical to fighting the war against chronic disease and to add healthy years to life. This seems particularly important for individuals who carry additional risk factors, such as type 2 diabetes mellitus, obesity, and the metabolic syndrome, and fail, as most do, to have a consistent and long-term weight loss.

\section{References}

[1] E. B. Rimm and M. J. Stampfer, "Diet, lifestyle, and longevity - the next steps?" Journal of the American Medical Association, vol. 292, no. 12, pp. 1490-1492, 2004.

[2] B. J. Willcox, Q. He, R. Chen et al., "Midlife risk factors and healthy survival in men," Journal of the American Medical Association, vol. 296, no. 19, pp. 2343-2350, 2006.

[3] L. B. Yates, L. Djoussé, T. Kurth, J. E. Buring, and J. M. Gaziano, "Exceptional longevity in men: modifiable factors associated with survival and function to age 90 years," Archives of Internal Medicine, vol. 168, no. 3, pp. 284-290, 2008.

[4] L. K. Heilbronn, L. de Jonge, M. I. Frisard et al., "Effect of 6-month calorie restriction on biomarkers of longevity, metabolic adaptation, and oxidative stress in overweight individuals: a randomized controlled trial," Journal of the American Medical Association, vol. 295, no. 13, pp. 1539-1548, 2006.

[5] R. Weindruch and R. S. Sohal, "Caloric intake and aging," New England Journal of Medicine, vol. 337, no. 14, pp. 986-994, 1997.

[6] E. J. Masoro, "Overview of caloric restriction and ageing," Mechanisms of Ageing and Development, vol. 126, no. 9, pp. 913-922, 2005.

[7] M. de Lorgeril, P. Salen, J. L. Martin, I. Monjaud, J. Delaye, and N. Mamelle, "Mediterranean diet, traditional risk factors, and the rate of cardiovascular complications after myocardial infarction: final report of the Lyon Diet Heart Study," Circulation, vol. 99, no. 6, pp. 779-785, 1999.

[8] R. B. Singh, G. Dubnov, M. A. Niaz et al., "Effect of an IndoMediterranean diet on progression of coronary artery disease in high risk patients (Indo-Mediterranean Diet Heart Study): a randomised single-blind trial," Lancet, vol. 360, no. 9344, pp. 1455-1461, 2002.

[9] K. Esposito and D. Giugliano, "Diet and inflammation: a link to metabolic and cardiovascular diseases," European Heart Journal, vol. 27, no. 1, pp. 15-20, 2006.

[10] D. Giugliano, A. Ceriello, and K. Esposito, "The effects of diet on inflammation. Emphasis on the metabolic syndrome," Journal of the American College of Cardiology, vol. 48, no. 4, pp. 677-685, 2006.

[11] K. Esposito, F. Giugliano, C. Di Palo et al., "Effect of lifestyle changes on erectile dysfunction in obese men: a randomized controlled trial," Journal of the American Medical Association, vol. 291, no. 24, pp. 2978-2984, 2004.

[12] K. Esposito, R. Marfella, M. Ciotola et al., "Effect of a Mediterranean-style diet on endothelial dysfunction and markers of vascular inflammation in the metabolic syndrome: a randomized trial," Journal of the American Medical Association, vol. 292, no. 12, pp. 1440-1446, 2004. 
[13] J. I. Cleeman, "Executive summary of the third report of the National Cholesterol Education Program (NCEP) expert panel on detection, evaluation, and treatment of high blood cholesterol in adults (adult treatment panel III)," Journal of the American Medical Association, vol. 285, no. 19, pp. 2486-2497, 2001.

[14] D. R. Matthews, J. P. Hosker, and A. S. Rudenski, "Homeostasis model assessment: insulin resistance and $\beta$-cell function from fasting plasma glucose and insulin concentrations in man," Diabetologia, vol. 28, no. 7, pp. 412-419, 1985.

[15] P. F. Bodary and D. T. Eitzman, "Adiponectin: vascular protection from the fat?" Arteriosclerosis, Thrombosis, and Vascular Biology, vol. 26, no. 2, pp. 235-236, 2006.

[16] J. D. Morrow, K. E. Hill, R. F. Burk, T. M. Nammour, K. F. Badr, and L. J. Roberts 2nd, "A series of prostaglandin Flike compounds are produced in vivo in humans by a noncyclooxygenase, free radical-catalyzed mechanism," Proceedings of the National Academy of Sciences of the United States of America, vol. 87, no. 23, pp. 9383-9387, 1990.

[17] J. D. Morrow, "Quantification of isoprostanes as indices of oxidant stress and the risk of atherosclerosis in humans," Arteriosclerosis, Thrombosis, and Vascular Biology, vol. 25, no. 2, pp. 279-286, 2005.

[18] E. Schwedhelm, A. Bartling, H. Lenzen et al., "Urinary 8-isoprostaglandin $\mathrm{F}$ as a risk marker in patients with coronary heart disease: a matched case-control study," Circulation, vol. 109, no. 7, pp. 843-848, 2004.

[19] J. F. Keaney, M. G. Larson, R. S. Vasan et al., "Obesity and systemic oxidative stress: clinical correlates of oxidative stress in the Framingham study," Arteriosclerosis, Thrombosis, and Vascular Biology, vol. 23, no. 3, pp. 434-439, 2003.

[20] J. B. Meigs, M. G. Larson, C. S. Fox, J. F. Keaney, R. S. Vasan, and E. J. Benjamin, "Association of oxidative stress, insulin resistance, and diabetes risk phenotypes: the Framingham Offspring Study," Diabetes Care, vol. 30, no. 10, pp. 2529-2535, 2007.

[21] K. B. Beckman and B. N. Ames, "The free radical theory of aging matures," Physiological Reviews, vol. 78, no. 2, pp. 547$581,1998$.

[22] M. Fitó, M. Guxens, D. Corella et al., "Effect of a traditional Mediterranean diet on lipoprotein oxidation: a randomized controlled trial," Archives of Internal Medicine, vol. 167, no. 11, pp. 1195-1203, 2007.

[23] A. Trichopoulou, T. Costacou, C. Bamia, and D. Trichopoulos, "Adherence to a Mediterranean diet and survival in a Greek population," New England Journal of Medicine, vol. 348, no. 26, pp. 2599-2608, 2003.

[24] K. T. B. Knoops, L. C. P. G. M. de Groot, D. Kromhout et al., "Mediterranean diet, lifestyle factors, and 10-year mortality in elderly European men and women: the HALE project," Journal of the American Medical Association, vol. 292, no. 12, pp. 14331439, 2004.

[25] C. Weyer, T. Funahashi, S. Tanaka et al., "Hypoadiponectinemia in obesity and type 2 diabetes: close association with insulin resistance and hyperinsulinemia," Journal of Clinical Endocrinology and Metabolism, vol. 86, no. 5, pp. 1930-1935, 2001.

[26] C. S. Mantzoros, C. J. Williams, J. E. Manson, J. B. Meigs, and F. B. Hu, "Adherence to the Mediterranean dietary pattern is positively associated with plasma adiponectin concentrations in diabetic women," American Journal of Clinical Nutrition, vol. 84, no. 2, pp. 328-335, 2006.
[27] J. A. Paniagua González, A. Gallego de la Sacristana, I. Romero et al., "Monounsaturated fat-rich diet prevents central body fat distribution and decreases postprandial adiponectin expression induced by a carbohydrate-rich diet in insulin-resistant subjects," Diabetes Care, vol. 30, no. 7, pp. 1717-1723, 2007. 


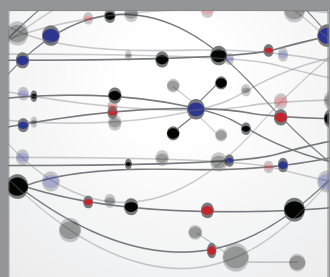

The Scientific World Journal
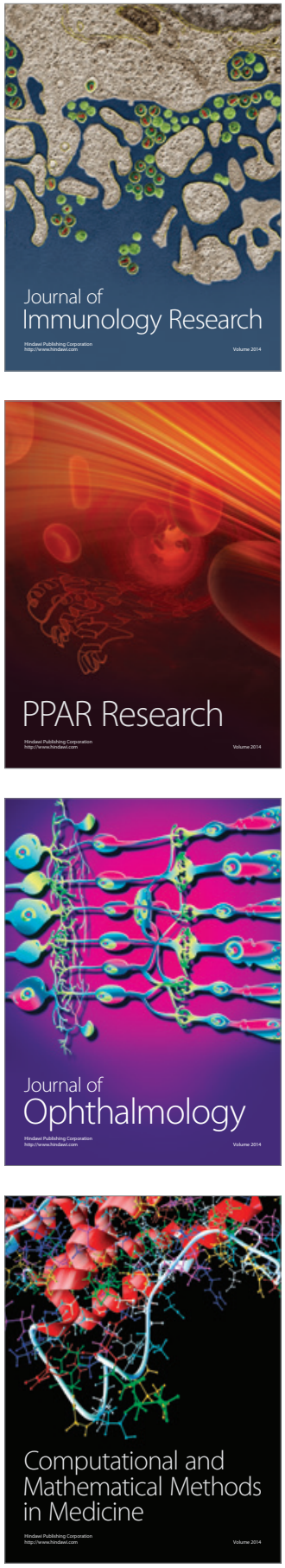

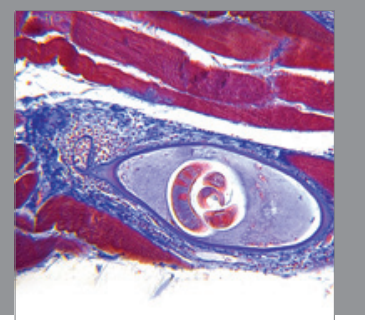

Gastroenterology

Research and Practice
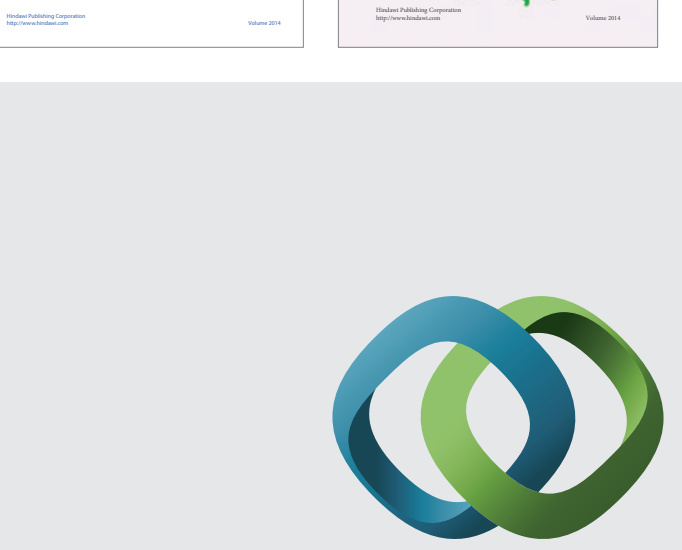

\section{Hindawi}

Submit your manuscripts at

http://www.hindawi.com
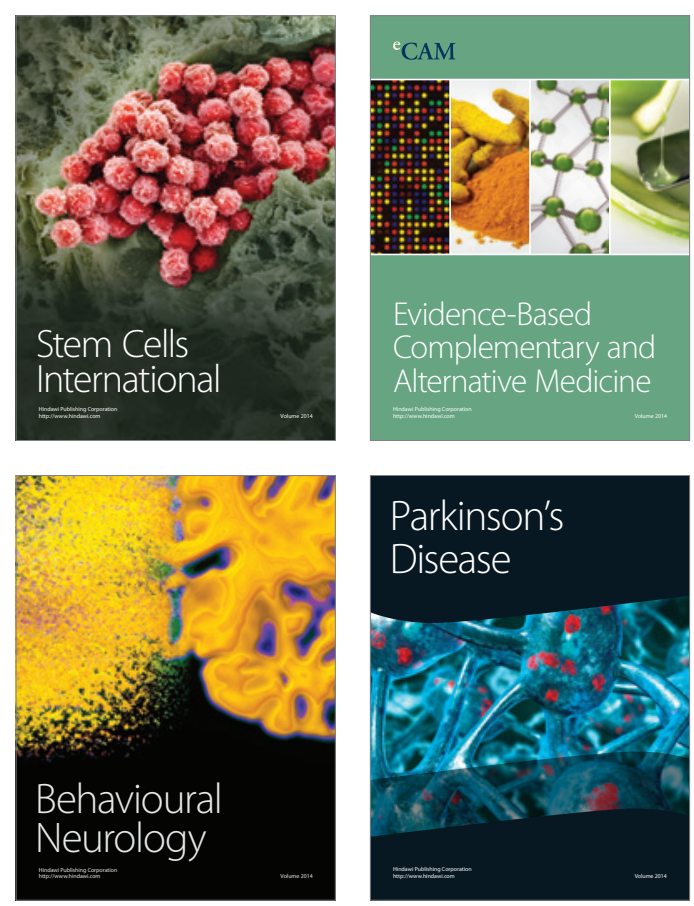

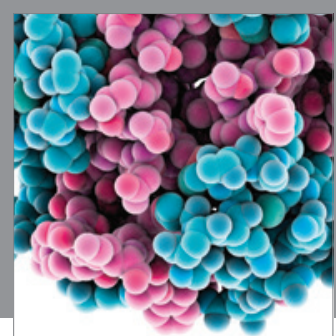

Journal of
Diabetes Research

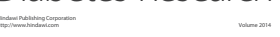

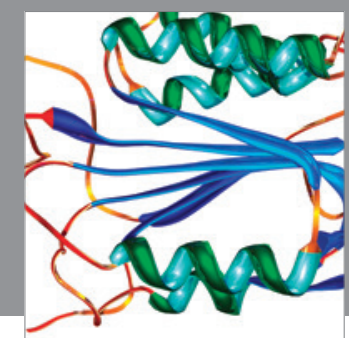

Disease Markers
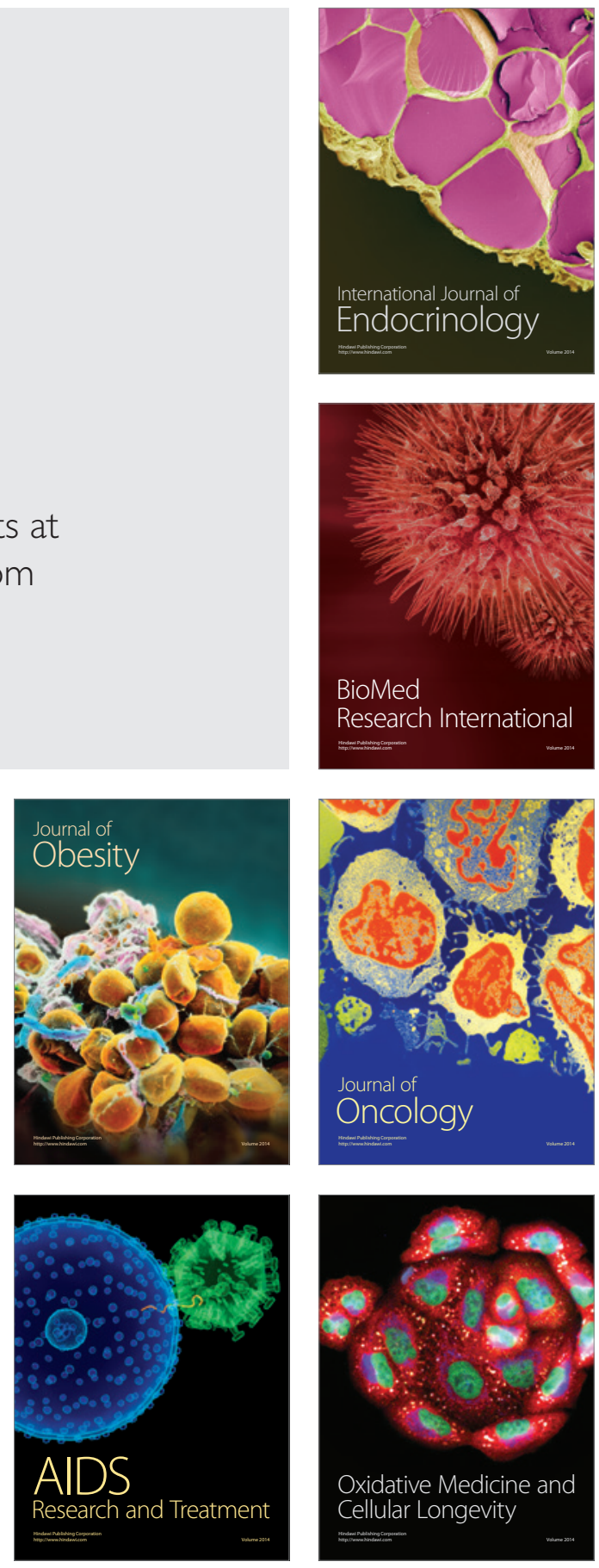